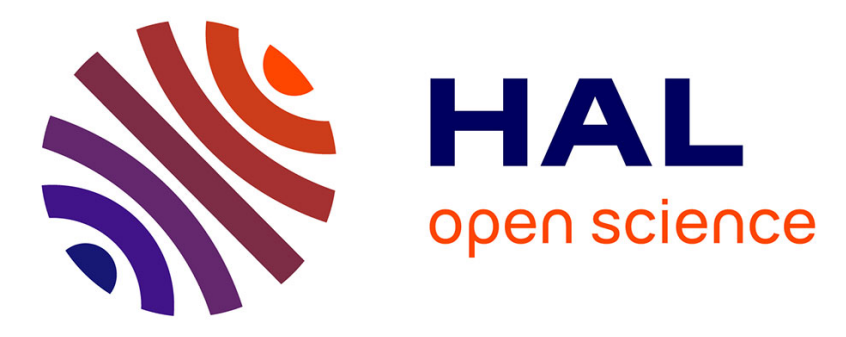

\title{
Modelling opinion formation by means of kinetic equations
}

\author{
Laurent Boudin, Francesco Salvarani
}

\section{To cite this version:}

Laurent Boudin, Francesco Salvarani. Modelling opinion formation by means of kinetic equations. Mathematical Modeling of Collective Behavior in Socio-Economic and Life Sciences, Springer, pp.245270, 2010, 10.1007/978-0-8176-4946-3_10. hal-00447659

\section{HAL Id: hal-00447659 \\ https://hal.science/hal-00447659}

Submitted on 15 Jan 2010

HAL is a multi-disciplinary open access archive for the deposit and dissemination of scientific research documents, whether they are published or not. The documents may come from teaching and research institutions in France or abroad, or from public or private research centers.
L'archive ouverte pluridisciplinaire HAL, est destinée au dépôt et à la diffusion de documents scientifiques de niveau recherche, publiés ou non, émanant des établissements d'enseignement et de recherche français ou étrangers, des laboratoires publics ou privés. 


\title{
Modelling opinion formation by means of kinetic equations
}

\author{
Laurent Boudin ${ }^{1,2,3}$ and Francesco Salvarani ${ }^{4}$
}

1 UPMC Univ Paris 06, UMR 7598 LJLL, Paris, F-75005, France -

laurent.boudin@upmc.fr

2 CNRS, UMR 7598 LJLL, Paris, F-75005, France

3 INRIA Paris-Rocquencourt, REO Project-team, F-78153 Le Chesnay Cedex, France

4 Dipartimento di Matematica F. Casorati, Università degli Studi di Pavia, Via Ferrata 1, 27100 Pavia, Italy - francesco.salvarani@unipv.it

Summary. In this chapter, we review some mechanisms of opinion dynamics that can be modelled by kinetic equations. Beside the sociological phenomenon of compromise, naturally linked to collisional operators of Boltzmann kind, many other aspects, already mentioned in the sociophysical literature or no, can enter in this framework. While describing some contributions appeared in the literature, we enlighten some mathematical tools of kinetic theory that can be useful in the context of sociophysics.

Key words: Sociophysics, opinion formation, kinetic theory

New opinions are always suspected, and usually opposed, without any other reason but because they are not already common.

John Locke, Essay on the Human Understanding

\section{Sociophysics}

\subsection{Introduction}

The success of statistical mechanics as a tool for describing physical systems composed by a great number of interacting elementary entities has induced some researchers to apply the same methodology to study problems of other sciences having the common feature that a global behaviour is obtained as a result of a chain of elementary processes. These new fields of application involve, for example, biology, economy and sociology. In particular, when describing a social phenomena, the basic entities are individuals, who interact with other members of the population by means of elementary mechanisms. 
The idea of using methods and concepts from physics to study sociological phenomena appeared in 1971 in a pioneering paper of Weidlich [79]. Some years later, Galam, Gefen and Shapir [38] first used the word sociophysics in order to characterize this kind of approach. They stated a global frame for sociophysics as a new field of research.

However, this strategy, even promising, is not exempted of conceptual difficulties. A major problem is that the individuals cannot be thought as simple mindless agents, and that their behaviour does not obey, in general, to some exact formulae. Human beings are indeed the opposite of simple elementary entities. The fine dynamics of their mental schemes is (and may remain) unknown. Moreover, in the real world, it is not guaranteed at all that two individuals, exactly stimulated in the same way, give the same answer.

Hence, a program based on the individuation of quantitative mechanisms in social dynamics could seem hopeless: modelling the behaviour of social agents by means of mathematical models implies an extreme simplification of the problem and, as a consequence, the inference of the macroscopic phenomenology starting from these basic models could seem without any predictive value. However, as observed in many applications of statistical physics, many qualitative (and sometimes quantitative) features of the system do not depend on the microscopic details of the processes under examination: only some high-level properties, such as conservation laws and symmetries, are important in order to obtain a coherent and reasonably correct description. Sociophysics is nowadays a recognized field research within statistical physics.

Beside the sociophysical approach, a good variety of other view points has been proposed to study the aforementioned questions. For example, whereas many papers adopt the point of view of mathematical statistics (for example, $[32,33,49,56])$, many other contributions are based on the language of game theory (among others, [44,64]) or fuzzy systems [24,66,67].

In what concern sociophysics, many problems have been studied and various approaches have been proposed. It is worth noting that, in the literature, the quantity of theoretical works is greater than empirical studies. The set of the most explored questions includes, among others, opinion and cultural dynamics, flocking, applause dynamics, hierarchies formation, human dynamics (including social web) and social networks. Because of its interest in the scientific community, a general overview on the research of social dynamics by means of a physical approach is available in some review articles. We quote $[20,37]$ and their references, that provide an outlook on different problems and methods.

\subsection{Towards the kinetic viewpoint of opinion formation}

Many strategies have been introduced in the literature to study the opinion formation. Some papers describe the phenomenon by using a discrete opinion variable, by working in the context of Ising models (for example, [38, 39, 73, 76]). An alternative strategy consists in describing opinion dynamics by using 
a continuous variable. Indeed, the opinion of an individual may smoothly evolve in a continuous space of possible opinions. For instance, the position of a social agent with a respect to a "yes/no" binary question can be expressed with nuance from the two extreme positions ("yes" and "no" without any doubt). An individual can also be indifferent regarding the question itself. In [26], the opinions lie in the closed interval $[0,1]$. The authors consider a population of $N$ agents, where each individual randomly interacts with one of his neighbours, following a mechanism close to a collisional mechanism in the Boltzmann equation with a cutoff effect. The population eventually reaches a stationary state, which can be a priori determined.

This collisional mechanism is not the only one that can be taken into account. As an example, in [3], the binary interaction process of [26] is combined with a diffusion one, which models the spontaneous changes of mind of the individuals. The competition between the two processes generates three possibilities. In absence of diffusion, the results from [26] are of course recovered and the concentration in some predefined opinions happens. When there is a weak diffusion, the favoured opinions again exist, but the concentration is not total. Eventually, when the diffusion is strong, the opinion becomes uniform in the population. Of course, there are many other phenomena which can be considered; some of them are discussed below.

We point out also that randomness is a key notion in the opinion formation process. As a matter of fact, the behaviours of the individuals can really randomly change, as well as the dynamics of the interaction between people. Moreover, the effects of external phenomena (such as, for example, mass media) are not necessarily fully predetermined.

Hence, any description of opinion formation dynamics must be able to consider random processes.

This consideration leads naturally to the kinetic approach, which is essentially the deterministic description of an underlying probabilistic phenomenon. It is based on a partial integrodifferential equation of Boltzmann type which governs the time evolution of an unknown function (normally a probability density) that describes the system.

Even if kinetic theory is an active area of research, however, a systematic specialised review describing the state-of-the-art concerning the kinetic approach in opinion dynamics is still missing. Goal of this chapter is to fill in the gaps in the existing literature. This review does not intend to cover all the question and approaches of sociophysics. In particular, the reader is also invited to read chapter ?? in the present book, which treats questions that are closely related to the contents of this contribution.

This work is divided into three parts. In the first one, we briefly present the Boltzmann equation, some of its mathematical properties and some way to discretize it. Then we point out the main phenomenon induced by the interaction mechanism between two social agents, the tendency to consensus. 
Eventually, we discuss other sociological phenomena which can be taken into account to compete with the natural compromise process.

\section{Kinetic approach in sociophysics: tools and methods}

The strategies, based on nonequilibrium statistical mechanics, which are used for modelling phenomena of opinion formation are mainly inspired to the classical field of the kinetic theory of rarefied gases. In this section, we briefly explain the basic aspects of the main examples of kinetic equation: the Boltzmann equation and its linear variant.

The purpose of both equations is to describe the time evolution of a system - composed by a great number of particles - by means of a distribution function in the phase space of the system, which can depend on a number of variables which is greater than the number of independent variables of the observables at a macroscopic scale.

We always assume that such a system is composed by identical particles, obeying the laws of classical mechanics, with only translational degrees of freedom. If the particles are contained in a domain $\Omega \subseteq \mathbb{R}^{3}$, the distribution function $f(x, v, t)$ of such a model should be defined on $\Omega \times \mathbb{R}^{3} \times \mathbb{R}^{+}$and, for all $t$, the integral

$$
\int_{x \in X} \int_{v \in V} f(x, v, t) \mathrm{d} x \mathrm{~d} v
$$

represents the number of particles contained in the space volume $X \subseteq \Omega$ with velocity in $V \subseteq \mathbb{R}^{3}$. Note that, in order to give a sense to the previous considerations, a reasonable hypothesis on $f$ is

$$
f(x, v, t) \in L_{\mathrm{loc}}^{1}\left(\Omega ; L^{1}\left(\mathbb{R}^{3}\right)\right), \quad \forall t \in \mathbb{R}^{+},
$$

(or, at least, $f(\cdot, \cdot, t)$ is a positive bounded measure on $K \times \mathbb{R}_{v}^{3}$, for every bounded subset $K$ of $\mathbb{R}_{x}^{3}$ ), which means that there is a finite number of particles in a bounded domain of the space. We shall here always consider, for the sake of simplicity, that $\Omega=\mathbb{R}^{3}$, and that the system is isolated, to avoid the effects of external forces on the particles.

If we suppose moreover that these particles do not mutually interact, then the time evolution of the distribution function $f$ is given by the free transport equation

$$
\frac{\partial f}{\partial t}+v \cdot \nabla_{x} f=0
$$

which means that the number of particles is conserved along the characteristics $\mathrm{d} x / \mathrm{d} t=v$ and $\mathrm{d} v / \mathrm{d} t=0$ (that is, $f(x, v, t)=f(x-v t, v, 0)$ ).

On the contrary, if the effect of the collisions between particles is no longer negligible, the description above does not hold, and one must also take into account these collisions. This leads, as we shall see, to add a non-vanishing second member in the free transport equation. 
In the next two subsections, we shall consider two situations: the Boltzmann equation and the linear transport equation. Then we provide some insights about the numerical methods which can be used for the Boltzmann equation.

\subsection{The Boltzmann equation}

If we suppose that the particles of the system interact through elastic and binary collisions, then the time evolution of $f$ is governed by the Boltzmann equation $[11,12]$ which, in the whole space and without external forces, has the following form:

$$
\frac{\partial f}{\partial t}+v \cdot \nabla_{x} f=Q^{+}(f, f)-f L(f)
$$

Here $Q_{+}$and $L$ are respectively a quadratic and a linear operator defined by

$$
Q^{+}(f, f)=\int_{\mathbb{R}^{3}} \int_{S^{2}} \sigma\left(v-v_{*}, \omega\right) f\left(x, v^{\prime}, t\right) f\left(x, v_{*}^{\prime}, t\right) \mathrm{d} \omega \mathrm{d} v_{*}
$$

and

$$
L(f)=\int_{\mathbb{R}^{3}} \int_{S^{2}} \sigma\left(v-v_{*}, \omega\right) f\left(x, v_{*}, t\right) \mathrm{d} \omega \mathrm{d} v_{*} .
$$

The parameter $\omega$ is a unit vector of the unit sphere $S^{2}$, so that $\mathrm{d} \omega$ is an element of area on the surface of the sphere, and $\left(v^{\prime}, v_{*}^{\prime}\right)$ are the pre-collisional velocities of two incident particles, related to the post-collisional velocities $\left(v, v_{*}\right)$ by the following relations:

$$
v^{\prime}=\frac{1}{2}\left(v+v_{*}+\left|v-v_{*}\right| \omega\right), \quad v_{*}^{\prime}=\frac{1}{2}\left(v+v_{*}-\left|v-v_{*}\right| \omega\right) .
$$

Moreover, the kernel $\sigma$ is a nonnegative function (or, at least, measure) which takes into account the details of the interactions between particles. It only depends on $\left|v-v_{*}\right|$ and on the scalar product $(k \cdot \omega)$, where $k=$ $\left(v-v_{*}\right) /\left|v-v_{*}\right|$.

The simplest case is when one deals with Maxwellian molecules. In this situation, the kernel $\sigma$ is reduced to a function of $(k \cdot \omega): \sigma\left(v-v_{*}, \omega\right)=\sigma(k \cdot \omega)$.

The spatially homogeneous Boltzmann equation (that is $\nabla_{x} f=0$ ) associated to such a kernel has several features: for example, as shown by Wild [81], it is possible to obtain a semi-explicit representation of the solution for the Cauchy problem of (1) under the form of a convergent series.

Kinetic equations (in particular, those in which appears a collision kernel) are usually derived (at the formal level) from Hamiltonian systems.

While, for linear equations, it is in general possible to give a rigorous proof of those derivations (see for example [41] for the linear Boltzmann equation), nonlinear equations are much more difficult to tackle, and rigorous results only 
exist for special regimes: in particular, for the Boltzmann equation, we mention the derivation of local (in time) solutions [22,58], or global (in time) small solutions $[50,51,71]$. When singular (nonlinear) kernels are concerned (non cutoff Boltzmann kernel, or Landau kernel), the derivation of the corresponding kinetic equation seems completely open, though the equations themselves have been extensively studied, and the only result obtained up to now, in that situation, is the kernel's characterization performed in [30], which allows to derive, starting from some physical abstract requirements, the collisional kernels of the kinetic equations but not the equations themselves.

Texts of reference on the wide subject of the Boltzmann equation, in which the arguments outlined above are more precisely discussed are $[21,22,78]$.

Finally, we note that there is a quantity of variants of (1). Among others, we recall Kac's model [55] and the discrete velocity models (see [42, 57, 65, 70] for additional information and bibliography).

\subsection{The linear transport equation for photons}

The behaviour of the distribution function for a set of photons in a medium can be governed by the linear transport equation. Even if it is simpler, this classical transport equation retains some features of the Boltzmann equation, among which the balance of gain and loss terms. The distribution function for photons $n=n(x, \nu, \omega, t)$ is defined on $\mathbb{R}^{3} \times \mathbb{R}^{+} \times S^{2} \times \mathbb{R}^{+}$. For all $t$, its integral

$$
\int_{x \in D} \int_{\nu \in\left[\nu_{0}, \nu_{1}\right]} \int_{\omega \in \Omega} n(x, \nu, \omega, t) \mathrm{d} x \mathrm{~d} \nu \mathrm{d} \omega
$$

represents the number of photons contained in the space volume $D \subseteq \mathbb{R}^{3}$, with frequency included in the interval $\left[\nu_{0}, \nu_{1}\right] \subseteq \mathbb{R}^{+}$and with velocity direction belonging to $\Omega \subseteq S^{2}$. The time evolution of the unknown $n$ is governed by a transport equation whose gain and loss terms are linear. In the whole space, the Cauchy problem for this equation has the following form:

$$
\frac{1}{c} \frac{\partial n}{\partial t}+\omega \cdot \nabla_{x} n=-\sigma(\nu) n+I(n)+S(\nu)
$$

with initial condition

$$
n(x, \nu, \omega, 0)=n_{0}(x, \nu, \omega) \quad(x, \nu, \omega) \in \mathbb{R}^{3} \times \mathbb{R}^{+} \times S^{2},
$$

where $\sigma(\nu)$ is the total cross section of absorption and scattering, depending on the frequency, $S(\nu)$ is a given source, $c$ is the speed of light and $I$ is a linear scattering operator defined by

$$
I(n)=\int_{\mathbb{R}^{+}} \int_{S^{2}} \frac{\nu}{\nu^{\prime}} \sigma_{s}\left(\nu^{\prime} \rightarrow \nu, \omega^{\prime} \cdot \omega\right) n\left(\nu^{\prime}, \omega^{\prime}\right) \mathrm{d} \nu^{\prime} \mathrm{d} \omega^{\prime} .
$$

Here $\nu^{\prime}$ and $\omega^{\prime}$ are respectively the pre-interaction frequency and velocity direction, and $\sigma_{s}$ is the scattering cross section, which represents the probability of a transition $\left(\nu^{\prime}, \omega^{\prime}\right) \mapsto(\nu, \omega)$. 
For a more extensive discussion we refer to the classical texts on the subject (in particular, $[19,23]$ ).

\subsection{Numerical methods}

The numerical solving of the Boltzmann equation (1) is ensured by a time split algorithm. During a time step one solves, on the one hand, the transport part, and, on the other hand, the collisional part. The latter part is usually the most expansive one, because of the collision operator nonlinearity.

There are mainly two ways to discretize the distribution function: the discrete velocity methods and the particle methods. One can find in the literature lots of improvement of the numerical methods briefly presented below, depending on the aims of the user: conserve some physical properties, asymptotical preserving schemes, etc.

The reader is invited to refer to [29] for more details.

\section{Particle method}

The distribution function is approximated by a sum of Dirac masses in the phase space $[27,28,74]$

$$
f(x, v, t)=\sum_{i} f_{i}(t) \delta_{X_{i}(t)}(x) \delta_{V_{i}(t)}(v),
$$

where $X_{i}(t)$ is the position of particle $i$ at time $t$, and $V_{i}(t)$ its velocity. The solving of the transport part is easy, since we just have to follow the particles along their trajectories. The discretization of the collisional operator is more intricate and is more often performed thanks to a Monte Carlo method, which induces a probabilistic treatment $[10,68,69]$. At the end of each collisional step, the locations and velocities of the particles have changed, but the quantity $f_{i}(t)$ has not. The collision process for the numerical particles mimics the behaviour of real physical particles, which ensures the conservation of physical quantities such that the momentum. Nevertheless, this kind of method generates a lot of computational noise. There are mainly two ways to decrease it: using a large number of particles or averaging numerical results.

\section{Discrete velocity method}

Deterministic methods can also be used to discretize the Boltzmann operator: the discrete velocity methods, see $[17,52,72]$ for example. The distribution function is given by its value on a uniform and time independent phase space grid. Formula (3) still holds, but this time, the positions and velocities do not change, only $f_{i}(t)$. But a couple of pre-collisional velocities generates a small number of possible post-collisional velocities. The mesh must then be very fine, and the computational cost may be very high [45]. 
In what concerns the models of opinion formation, the choice of the numerical method widely depends on the phenomena taken into account, and the relevance of each method must therefore be studied. Nevertheless, the time splitting should still be considered since, in most situations, one deals with several different phenomena, with specific modelling features.

\section{The main phenomenon: the compromise}

As we already pointed out, the feature which appears in almost all models of opinion formation is the tendency to compromise, which mimics the collisions happening in the traditional Boltzmann equation. That means that the binary interactions naturally tend to concentrate the opinions of the population around some values (the average one, or, in other cases, periodic values, for instance).

\subsection{Basic models}

\section{Towards continuous models}

The introduction of kinetic models in the context of sociophysics goes back to the beginning of the nineties. In [47], Helbing points out that the master equation and Boltzmann-like equations are suitable for the quantitative description of behaviour changes and social processes.

In the aforementioned article, the author does not limit himself to consider problems of opinion formation, but introduces a general framework for social situations described by a system of $N$ individuals, whose state $y \in\{1, \ldots, S\}$ represents the possible behaviour strategies concerning a certain situation. It is quite clear that the first Helbing model is a discrete velocity model. Note that this kind of model have been used later on to study opinion dynamics phenomena (for example, [6-9]). However, in the absence of spatial phenomena, their structure more remembers a dynamical system than a kinetic model. We therefore invite the interested reader to directly refer to the literature.

The dynamics of the system is then described by a master equation, which is a phenomenological set of first-order differential equations describing the time evolution of the probability of a system to occupy each one of a discrete set of states.

If we denote by $n_{y}$ the number of subsystems in state $y$, which must satisfy the constraint

$$
\sum_{y} n_{y}=N
$$

by $\boldsymbol{n}=\left(\cdots, n_{y}, \cdots\right)$ the configuration vector of the system and by $P(\boldsymbol{n}, t)$ the probability of observing the configuration $\boldsymbol{n}$ at time $t$, the master equation of the system is 


$$
\frac{\mathrm{d} P}{\mathrm{~d} t}(\boldsymbol{n}, t)=\sum_{\boldsymbol{n}^{\prime}} w\left(\boldsymbol{n} \mid \boldsymbol{n}^{\prime} ; t\right) P\left(\boldsymbol{n}^{\prime}, t\right)-\sum_{\boldsymbol{n}^{\prime}} w\left(\boldsymbol{n}^{\prime} \mid \boldsymbol{n} ; t\right) P(\boldsymbol{n}, t),
$$

where $w\left(\boldsymbol{n}^{\prime} \mid \boldsymbol{n} ; t\right)$ are the configurational transition rates from configuration $\boldsymbol{n}$ to configuration $\boldsymbol{n}^{\prime}$.

There are two types of transitions: spontaneous changes of state of a single subsystem, or a direct pair interaction. The form of the transition rates $w\left(\boldsymbol{n}^{\prime} \mid \boldsymbol{n} ; t\right)$ is

$$
w\left(\boldsymbol{n}^{\prime} \mid \boldsymbol{n} ; t\right)=w_{1}\left(y^{\prime} \mid y ; t\right) n_{y}
$$

if $\boldsymbol{n}^{\prime}=\left(\cdots,\left(n_{y^{\prime}}+1\right), \cdots,\left(n_{y}-1\right), \cdots\right)$, in the case of spontaneous transitions,

$$
w\left(\boldsymbol{n}^{\prime} \mid \boldsymbol{n} ; t\right)=w_{2}\left(y^{\prime}, z^{\prime} \mid y, z ; t\right) n_{y} n_{z}
$$

if $\boldsymbol{n}^{\prime}=\left(\cdots,\left(n_{y^{\prime}}+1\right), \cdots,\left(n_{y}-1\right), \cdots,\left(n_{z^{\prime}}+1\right), \cdots,\left(n_{z}-1\right), \cdots\right)$, in the case of binary interactions, and

$$
w\left(\boldsymbol{n}^{\prime} \mid \boldsymbol{n} ; t\right)=0
$$

otherwise.

Under this situation, and in absence of spontaneous transitions, the master equation reduces to be an homogeneous discrete velocity model with quadratic collisional part, whose quadratic structure is a discrete version of the collisional integral of a Boltzmann equation.

The states of the system are strategies of individuals playing a game with others, which they randomly meet. As a result of these collisions, they change their strategies by adopting those of their more successful opponents with probabilities proportional to the difference between the expected successes of the latter and their own.

The author classifies the pair interactions in four types, denoted as (I), (II), (III), (IV), whose interpretation is the following.

1. Interactions (I) describe imitative processes, that is the tendency to take over the strategy of another individual.

2. Interactions (II) describe avoidance processes, where an individual changes the strategy when meeting another individual using the same strategy (processes of this kind are known as aversive behaviour, defiant behaviour or snob effect).

3. Interactions (III) represent some kind of compromising processes, where an individual changes the strategy to a new one (the "compromise") when meeting an individual with another strategy (such processes are found, if a certain strategy cannot be maintained when confronted with another strategy).

4. Interactions (IV) describe another kind of imitative processes, different from processes of type (I): an individual changes the strategy despite the fact that he convinces his interaction partner of the strength of his strategy. Social processes of this kind are very improbable and can usually be neglected. 
Moreover, the author studies the behaviour of the most probable strategy distribution of the previous model and shows that its time evolution is governed by a Boltzmann-like equation.

In [46], the author deeply explores the properties of the master equation (4), and shows that it is consistent with many models of social theory, e.g. the diffusion models, Lewin's field theory, the logistic equation, the gravity model, the Weidlich-Haag model, or the game dynamical equations.

Assuming that the set $\Omega$ of possible behaviours $\boldsymbol{x}$ is continuous, the master equation (4) is reformulated as a space-homogeneous Boltzmann equation

$$
\frac{\partial P}{\partial t}(\boldsymbol{x}, t)=\int_{\Omega}\left[w\left(\boldsymbol{x} \mid \boldsymbol{x}^{\prime} ; t\right) P\left(\boldsymbol{x}^{\prime}, t\right)-w\left(\boldsymbol{x}^{\prime} \mid \boldsymbol{x} ; t\right) P(\boldsymbol{x}, t)\right] \mathrm{d} \boldsymbol{x}^{\prime} .
$$

A Kramers-Moyal expansion (which is essentially a second order Taylor approximation) of (5) leads to the Boltzmann-Fokker-Planck equation

$$
\frac{\partial P}{\partial t}=-\sum_{i=1}^{n} \frac{\partial\left(K_{i} P\right)}{\partial x_{i}}+\frac{1}{2} \sum_{i, j=1}^{n} \frac{\partial^{2}\left(Q_{i j} P\right)}{\partial x_{i} \partial x_{j}}
$$

where

$$
K_{i}(\boldsymbol{x}, t)=\int_{\Omega}\left(x_{i}^{\prime}-x_{i}\right) w\left(\boldsymbol{x}^{\prime} \mid \boldsymbol{x} ; t\right) \mathrm{d} \boldsymbol{x}^{\prime}, \quad 1 \leq i \leq n,
$$

are the effective drift coefficients and

$$
Q_{i j}(\boldsymbol{x}, t)=\int_{\Omega}\left(x_{i}^{\prime}-x_{i}\right)\left(x_{j}^{\prime}-x_{j}\right) w\left(\boldsymbol{x}^{\prime} \mid \boldsymbol{x} ; t\right) \mathrm{d} \boldsymbol{x}^{\prime} \quad 1 \leq i, j \leq n,
$$

are the effective diffusion coefficients.

Whereas the drift coefficients govern the systematic change of the distribution $P(\boldsymbol{x}, t)$, the diffusion coefficients describe its spreading, due to fluctuations resulting from the individual variation of behaviour changes.

This formulation allows to introduce two concepts: the social forces and the social fields. We only remark here that a social field represents, in the model, the influence of the public opinion, social constraints and tendencies.

In [48], a method for solving the master equation (4) is presented, by writing the unknown $P$ in an approximate form. The exact solution can then be obtained as an approximate expression of a path integral.

\section{Social state for an electoral competition}

In his works [60-63], Lo Schiavo develops kinetic models of Boltzmann type for social dynamics, to eventually reach a relevant description of an electoral competition. The first model he used [60] is an adapted version of the Jäger and Segel population dynamics model [53]. It is fitted to describe interacting agents who are characterized by a continuous variable $u \in \mathbb{R}$, called the social 
state (poor/rich). It opened the road to model an electoral competition in his following articles.

Note that the model of [60] is close to Helbing's, since the variable of the density function is not yet the opinion, but a social state, which is quite similar to the configuration vector $\boldsymbol{n}$ in [46-48].

In [61], the distribution functions describing the population are modified by two kinds of phenomena: the collisions between individuals, which are an internal process, and external forces. More precisely, the population is divided into groups, e.g. political parties, and the agents can choose their belonging to one group or another, or not belonging to any. The population inside each party is then described by a specific distribution function. The external forces can obviously have an effect each distribution function. They also allow the transition between groups by somehow influencing the choice of each individual with respect to the groups, i.e. the mass exchange between each group related distribution function can happen.

Let us give some more details on the model. We denote by $p$ the number of available groups. The variable of the distribution functions this time lies in $[-1,1]$. It is not related to a specific group, it only represents the feeling of an agent about his social condition and his opinion with regard to the society where he lives. The positive values of $u$ mean that the associated individual is quite happy, and the negative ones mean he is unsatisfied. If the collision process inside each group is standard, Lo Schiavo also defines another interaction process between individuals with different states and groups. Like in [2], the kinetic system that rises from these assumptions reads, for any $1 \leq i \leq p$,

$$
\frac{\partial f_{i}}{\partial t}+\frac{\partial\left(f_{i} K_{i}(\boldsymbol{f})\right)}{\partial u}=Q_{i}^{+}(\boldsymbol{f})-Q_{i}^{-}(\boldsymbol{f}),
$$

where each $f_{i}$ is the distribution function for the $i^{\text {th }}$ party, $\boldsymbol{f}=\left(f_{i}\right)_{1 \leq i \leq p}$, $Q_{i}^{+}(\boldsymbol{f})$ and $Q_{i}^{-}(\boldsymbol{f})$ are respectively the gain and loss terms for the $i^{\text {th }}$ party due to the collisional processes, and eventually $K_{i}(\boldsymbol{f})$ is a propaganda operator, which draws an individual from a given party to the $i^{\text {th }}$ party. Note that this propaganda function is very similar to the social force defined by Helbing in [48]. In the previous operators, some frequency functions are used to drive the probability for which each kind of interaction occurs. Those frequencies allow to describe the asymptotic behaviour of the model, see 3.2 .

\section{Fully collisional model}

Since the main phenomenon in the opinion formation process is the compromise, it is quite natural to investigate a model where only collisional effects happen. We mainly focus now on systems where the variable of the distribution function is the opinion itself. It is systematically denoted with the letter $x$ in the remaining of the chapter.

Ben-Naim et al. $[4,5]$ use a very similar microscopic model to the one from [26], to study the opinion dynamics, in a situation when the individuals 
reach compromise through binary interactions. They assume that the opinions of the population lie in a closed interval $[-\Delta,+\Delta], \Delta>0$. When the opinions $\left(x_{1}, x_{2}\right)$ of two individuals are close enough, in that case, $\left|x_{2}-x_{1}\right|<1$, they both acquire the average opinion $\left(x_{1}+x_{2}\right) / 2$. Otherwise, there is no interaction. The density function of the population $f$ satisfies the following rate equation of Boltzmann type:

$$
\begin{aligned}
\frac{\partial f}{\partial t}(x, t)=\int_{\left|x_{*}-x\right|<1 / 2} f\left(x_{*}, t\right) f\left(2 x-x_{*}, t\right) \mathrm{d} x_{*} \\
\quad-\int_{\left|x_{*}-x\right|<1} f(x, t) f\left(\left(x+x_{*}\right) / 2, t\right) \mathrm{d} x_{*},
\end{aligned}
$$

supplemented by the uniform initial condition $f(x, 0)=1$ for any $x \in$ $[-\Delta,+\Delta]$. We recover in (7) the form of the collision kernel, with a gain term and a loss one. It is quite clear that the first two moments of $f$ are conserved. When $\Delta<1 / 2,(7)$ can be integrated, and one can prove that the distribution function asymptotically reaches an equilibrium, which is a Dirac mass in 0 , weighted by the (constant) moment of $f$ of order 0 .

When $\Delta \geq 1 / 2$, the system reaches a steady state with a finite number of isolated, non-interacting opinion clusters. More precisely, the asymptotic distribution function is a weighted linear combination of Dirac masses. The weights must obviously satisfy the mass and momentum conservations. When $\Delta$ grows, the number of the clusters undergoes a periodic sequence of bifurcations. Both strong (weight $>1$ ) and weak (weight $<10^{-2}$ ) clusters appear, and they are organized in alternating pattern. Moreover, the period of the bifurcations govern the total number of clusters. If $\Delta \gg 1$, there are $4 \Delta / L$ clusters appearing.

Note that, in [5], the authors also present a simplified and discretized version of the latter model, where each individual has only three possible opinion: left, centre and right. The range of interaction is then translated, in that situation, by the fact that the people on the left and the right sides cannot interact. It then results in everyone either with a centred opinion, or with no people at the centre at all.

\section{Compromise versus self-thinking}

As we shall detail in Section 4, the opinion dynamics come from the competition between several phenomena. Nevertheless, we immediately present models where this kind of competition takes place. Indeed, it allows to present specific collisional rules, which are really studied in the articles of interest, and set the basis for the majority of the following articles.

The first works of Toscani [77] and Boudin and Salvarani [15] on the opinion formation are directly inspired from [3] in a kinetic formulation. Indeed, they both involve two phenomena: the binary interactions between individuals and the diffusion process, which models the possible spontaneous change of 
opinion of each individual, i.e. the self-thinking. The opinion variable $x$ lies in $[-1,+1]$, where, once again, $(-1)$ and $(+1)$ denote the extreme opposite opinions. They also both provide specific collision rules. The main difference between the two models lies in the fact that the diffusion process is inserted in the collision rules in [77], whereas, in [15], it is taken into account in the kinetic equation.

In [77], the collision rules read

$$
\begin{aligned}
x^{\prime} & =x-\gamma P(|x|)\left(x-x_{*}+\eta D(|x|)\right), \\
x_{*}^{\prime} & =x_{*}-\gamma P\left(\left|x_{*}\right|\right)\left(x_{*}-x+\eta_{*} D\left(\left|x_{*}\right|\right)\right),
\end{aligned}
$$

where $x$ and $x_{*}$ are the pre-collisional opinions, and $x^{\prime}$ and $x_{*}^{\prime}$ the postcollisional ones. In (8)-(9), $\gamma$ is a constant such that $0<\gamma<1 / 2$ which describes the tendency to consensus. The random variables $\eta$ and $\eta_{*}$, whose values lie in a subset $\mathcal{H}$ of $\mathbb{R}$, have the same distribution, with zero mean and variance $\sigma^{2}$, where $\sigma$ is a parameter which characterizes the self-thinking. Eventually, $P$ and $D$ model the local (in the opinion variable) influence of each phenomenon.

Then the distribution function once again solves the following kinetic equation of Boltzmann type:

$$
\frac{\partial f}{\partial t}(x, t)=\int_{\mathcal{H}^{2}} \int_{-1}^{1}\left({ }^{\prime} \beta \frac{1}{J} f\left({ }^{\prime} x, t\right) f\left({ }^{\prime} x_{*}, t\right)-\beta f(x, t) f\left(x_{*}, t\right)\right) \mathrm{d} x_{*} \mathrm{~d} \eta \mathrm{d} \eta_{*},
$$

where ' $x$ and ' $x_{*}$ are the pre-collisional opinions which give, after interaction, the opinions $x$ and $x_{*}$. The function $\beta$ describes the interaction rate, and $J$ is the Jacobian of the collision rules (8)-(9).

In [15], the pre-collisional opinions $x$ and $x_{*}$ are changed in the following way:

$$
\begin{aligned}
x^{\prime} & =\frac{x+x_{*}}{2}+\eta(x) \frac{x-x_{*}}{2}, \\
x_{*}^{\prime} & =\frac{x+x_{*}}{2}+\eta\left(x_{*}\right) \frac{x_{*}-x}{2},
\end{aligned}
$$

where $x^{\prime}$ and $x_{*}^{\prime}$ are the post-collisional opinions. The function $\eta$ is an attraction coefficient: in general, it is a smooth function which describes the degree of attraction of the average opinion with respect to the starting opinion of the agent. The collision mechanism (11)-(12) translates the fact that a strong opinion is less attracted towards the average opinion than a weaker one.

The self-thinking is taken into account as a global term in the kinetic equation, which then reads

$$
\frac{\partial f}{\partial t}=\frac{\partial}{\partial x}\left(\alpha \frac{\partial f}{\partial x}\right)+Q(f, f)
$$

The function $\alpha$ only depends on the opinion variable, and holds the information that a strong opinion is more stable than a weaker one. The collision 
kernel $Q(f, f)$ can easily be written under a weak form. Let $\phi=\phi(x)$ a suitable test function. We have

$$
\langle Q(f, f), \varphi\rangle=\beta \iint_{(-1,1)^{2}} f(t, x) f\left(t, x_{*}\right)\left[\varphi\left(x^{\prime}\right)-\varphi(x)\right] \mathrm{d} x_{*} \mathrm{~d} x .
$$

Once again, $\beta>0$ describes the interaction rate, but is set to a constant value at the beginning.

In both papers, some mathematical properties of the model are discussed. The discussion of [77] is focused on the moments of $f$ to prepare for the study of the quasi-invariant limit, see 3.2. In [15], the authors prove the existence of the solution to (13)-(14), with an initial datum in $L^{1}(-1,1)$, lying in $L^{\infty}\left(0, T ; L^{1}(-1,1)\right)$, for any $T>0$.

\subsection{Mean field approximation}

When one considers a kinetic system with lots of interacting particles, it is most often very difficult to obtain an exact solution, except for very simple cases, such as the one-dimensional Ising model, for instance. The main idea, here, is to replace the microscopic interactions by a unique averaged interaction implying, most of the time, moments of the distribution function. This reduces the kinetic system into a simplified problem. The average behaviour of the kinetic system can then be obtained in an easier way.

Consequently, we can also get more easily the time asymptotic behaviour of the distribution function, which is relevant, because it helps to quite accurately describe the stationary solutions of the kinetic equation. In the kinetic framework, the asymptotic models are often quite simple, for example, of Fokker-Planck type.

In the final part of [61], Lo Schiavo discusses, with a computational point of view, the asymptotic behaviour of his basic and extended (with external forces) models. He points out that this behaviour mainly depends on the various frequencies of interaction used in the models.

\section{The quasi-invariant limit}

The studies from [77] and [16] tackle the question of the quasi-invariant limit from both mathematical and numerical points of view. They both assume that the collisional and diffusive effects are small, but remain linked.

Boudin and Salvarani [16] assume that their attraction function $\eta$ used in (11)-(12) is a constant close to 1, i.e. $\eta=1-\varepsilon, \varepsilon>0$, and that the diffusion coefficient $\alpha$ in (13) has the form $\alpha(x)=\varepsilon^{k} \alpha_{0}(x)$ for any $x$. They discuss the limit, when $\varepsilon$ goes to $0^{+}$, of the distribution function, with respect to the parameter $k$. They derive three different regimes: the collision-dominated one $(k>1)$, the diffusion-dominated one $(k<1)$ and the equilibrated one $(k=1)$, where the collision kernel is replaced by both linear and nonlinear (because of 
a moment of $f$ ) terms. In the first case, they obtain an exact solution of the limit equation, which goes to a Dirac mass, when the time grows. In the second one, they provide an estimate of the convergence speed to the equilibrium of the solution to the the limit equation, the existence of which was previously obtained in [18]. Eventually, in the third case, they study the steady states and obtain, under some assumptions on $\beta$, an exponential rate of convergence.

Toscani [77] shows that the constants $\gamma$ and $\sigma$ involved in (8)-(9) are the key quantities to remember the microscopic collision mechanism when passing to the quasi-invariant limit (i.e. when $\gamma$ and $\sigma$ both go to 0 ), through the ratio $\lambda=\sigma^{2} / \gamma$. In fact, he recovers the three same regimes as in [16] with respect to the value of $\lambda\left(0\right.$, in $\mathbb{R}_{+}^{*}$ or $\left.+\infty\right)$. In the equilibrated regime, i.e. when $\lambda>0$, he obtains the convergence of the time scaled weak solutions $f(x, \tau / \gamma)$ to $(10)$, towards a function $g(x, \tau)$, which is a weak solution to the following Fokker-Planck equation

$$
\frac{\partial g}{\partial \tau}=\frac{\lambda}{2} \frac{\partial^{2}}{\partial x^{2}}\left(D(|x|)^{2} g\right)+\frac{\partial}{\partial x}((x-m) g),
$$

where

$$
m=\int_{-1}^{1} x f(x, 0) \mathrm{d} x
$$

is the initial mean opinion. Note that it is really important here to assume that the interaction rate $\beta$ does not depend on the opinion, and that $P=1$. For the other regimes, he also introduces a suitable asymptotic limit of the model yielding a Fokker-Planck equation.

\section{An example}

Aletti, Naldi and Toscani [1] study one of these Fokker-Planck equations:

$$
\frac{\partial f}{\partial t}=\gamma \frac{\partial}{\partial x}\left(\left(1-x^{2}\right)(x-m(t)) f\right),
$$

where $\gamma$ is linked to the spreading $(\gamma=-1)$ or the concentration $(\gamma=1)$ of the opinions. For $\gamma=1$, equation (16) directly comes from the asymptotic limit in the collision-dominated case in [77], choosing $P(y)=1-y^{2}$. For $\gamma=-1$, it looks like the model presented in [73], obtained by a mean field approximation of the Sznajd model [76], in the case of two opinions.

Unlike (15), (16) is really nonlinear, because the time depending mean opinion is involved:

$$
m(t)=\int_{-1}^{1} x f(x, t) \mathrm{d} x .
$$

They use some properties of a pseudoinverse of the cumulative distribution function to obtain the well-posedness of their problem, and the existence and uniqueness of solutions. Then they derive results on their large-time behaviour. The two values of the parameter $\gamma$ call for separate treatments. In 
the spreading case, they prove that the limit distribution function is given by two masses located in \pm 1 . In the concentration case, the limit has one or three Dirac masses, and in the latter case, two of them are located in \pm 1 and appear because they already exist at initial time.

\section{Other sociological phenomena}

Up to now, we pointed lout one main phenomenon in the opinion formation process, the compromise. We even add for some basic models the self-thinking process, which appears either in the collision mechanism [1,77], or in the kinetic equation itself $[15,16]$. In fact, there are obviously lots of sociological phenomena which can be a part of the competition with the collisional process. We only present some of them here, because they appeared in the kinetic literature. The reader can refer to the review articles on the topic $[20,37]$ to find other tracks.

\subsection{From the opinion to the choice}

The question of the choice/vote naturally rises after the opinion formation. Indeed, when someone votes, he does not necessarily only follow his own opinion, he may try to prevent the electoral results to be too far away from his opinion. It is quite clear that a vote model simultaneously depends on the voter opinion and on the electoral system. In the sociophysical literature, voting models are provided, for instance, in $[40,59,75]$.

Comincioli, Della Croce and Toscani [25] propose a possible approach to the formation of choice. They mostly follow [77], but they add a fixed distribution of possible choices $M(x)$, which can be seen, in the kinetic theory, as a fixed background of field particles. Since they only consider opinions regarding a finite number $N+1$ of questions, they can write a typical form of the background:

$$
M(x)=\sum_{0}^{N} \omega_{i} \delta_{\bar{x}_{i}}(x), \quad \sum_{0}^{N} \omega_{i}=1 .
$$

Let us give some explanations about the coefficients in (17). The parameters $\omega_{i} \in(0,1)$ are the probability that an agent chooses the $i^{\text {th }}$ possibility. The values $\bar{x}_{i} \in(-1,1) \backslash\{0\}$ represent the stability of the $i^{\text {th }}$ choice. They reflect the fact that extreme opinions are more difficult to change.

The population and the background interact, and it is translated in a microscopic collision rule by

$$
\left.x^{\prime}=x-\gamma P\left(\left|x-x_{*}\right|\right)\left(x-x_{*}\right)+\eta D\left(\left|x^{2}\right|\right)\right),
$$

which is very similar to (8). The functions $P$ and $D$, the random variable $\eta$ and the constant $\gamma$ have the same meaning as in [77]. 
The time evolution of the distribution function $f$ is described by a kinetic equation involving a linear collisional integral of Boltzmann type. It reads

$$
\frac{\partial f}{\partial t}=Q(f, M)
$$

where the collisional operator can be written in its weak form, for any smooth enough test function $\phi$, as

$$
\langle Q(f, M), \phi\rangle=\iint_{(-1,1)^{2}} f(x, t) M\left(x_{*}\right)\left(\phi\left(x^{\prime}\right)-\phi(x)\right) \mathrm{d} x \mathrm{~d} x_{*} .
$$

Then they tackle the quasi-invariant limit of their system (19)-(20). The linear Boltzmann equation is again asymptotically well described by a FokkerPlanck type equation. This Fokker-Planck type equation recovers and generalizes analogous one obtained by mean field approximation of the voter model in [75].

\subsection{Contradictory individuals}

In $[34,36]$, Galam introduces and uses the notion of "contrarian" people to explain some major recent electoral phenomena. That kind of individuals cannot be convinced by standard arguments. In fact, they may systematically oppose the majority opinion, whatever it is.

In [13], the authors introduce the notion, a little bit different, of contradictory people, opposed to conciliatory ones. When interacting with conciliatory individuals, who tend to compromise, they follow the opposite microscopic opinion, instead of simply going away from the average one.

More precisely, if they still consider conciliatory people who tend to compromise, they also take into account two kinds of interactions involving contradictory people. Let $x$ denote the opinion of a conciliatory individual and $x_{*}$ the opinion of a contradictory one, before interaction, and $x^{\prime}, x_{*}^{\prime}$ the respective post-collisional opinions. The first type of interaction writes

$$
\begin{aligned}
x^{\prime} & =\frac{x+x_{*}}{2}+\eta(x) \frac{x-x_{*}}{2}, \\
x_{*}^{\prime} & =-\alpha\left(x_{*}\right)\left[\frac{x_{*}+x}{2}+\eta\left(x_{*}\right) \frac{x_{*}-x}{2}\right] .
\end{aligned}
$$

If (21) is still the same as in [15], the opinion of the contradictory individual uses the standard post-collisional opinion and shifts it to an opposite value using a reaction function $\alpha$.

In the second kind of interaction, the value of $x^{\prime}$ is the same as in (21), but the new value of $x_{*}^{\prime}$ is given by 


$$
x_{*}^{\prime}= \begin{cases}1-\frac{\left(1-x^{\prime}\right)\left(1-x_{*}\right)}{(1-x)} & \text { if } x<x_{*}, \\ x_{*} & \text { if } x=x_{*}, \\ \frac{\left(1+x^{\prime}\right)\left(1+x_{*}\right)}{(1+x)}-1 & \text { if } x>x_{*} .\end{cases}
$$

This time, the contradictory opinion tends to go to \pm 1 , just to stay away from the conciliatory one it interacted with.

For each kind of interaction, they also define the contradictory-contradictory collision mechanism.

In any case, the kinetic system satisfied by $f$ and $g$ is the following:

$$
\begin{aligned}
& \frac{\partial f}{\partial t}=Q(f, f)+R_{1}(f, g), \\
& \frac{\partial g}{\partial t}=R_{2}(f, g)+S(g, g),
\end{aligned}
$$

where $Q(f, f)$ is the collision kernel associated to the interaction between conciliatory people, $R_{1}(f, g)$ and $R_{2}(f, g)$ are the kernels for the mixed conciliatory-contradictory interaction, and $S(g, g)$ is the collision kernel associated to the interaction between contradictory people. The authors obtain an existence result on $f$ and $g$ in $L^{\infty}\left(0, T ; L^{1}(-1,1)\right)$.

They next numerically investigate the asymptotic behaviour of the solutions of (24)-(25). If they use (21)-(22) as collision rules, both $f$ and $g$ go to the Dirac mass centred at 0 , and if they use (23) instead of (22), they obtain two Dirac masses in \pm 1 for $g$, but not necessarily well-balanced, a concentration of $f$ around an opinion which changes periodically in time. This latter behaviour must be underlined, because it means that there is eventually no steady state for $f$.

\subsection{Leadership}

The concept of leadership is a key point of sociology and many authors have investigated its role in various aspects of the society. For example, many studies recognize that leaders have a crucial role in establishing the organization of pyramidal hierarchies, including large corporations, universities, armies, trade unions or political parties (see, for example, [35]).

In [31], the authors explain the formation of opinions in a society by supposing that there exist two categories of people: the opinion leaders (group 2), who are active media users that select, interpret, modify, facilitate and transmit the information, and the less active part of the population (group 1), more passive and ductile. The individuals of the population interact between themselves and modify their opinions by means of a collisional rule which is a variant of Toscani's [77].

In fact, as in [77], the opinion variable $x$ lies in $[-1,1]$. Both groups are described by distribution functions $\left(f_{i}\right)_{i=1,2}$. If two individuals from the same 
group $i$ discuss, the post-collisional opinions $x^{\prime}, x_{*}^{\prime}$ generated by the interaction of individuals with pre-collisional opinions $x, x_{*}$ are obtained through the following formulae:

$$
\begin{aligned}
x^{\prime} & =x-\gamma_{i} P_{i}\left(\left|x-x_{*}\right|\right)\left(x-x_{*}\right)+\eta_{i 1} D_{i}(x), \\
x_{*}^{\prime} & =x_{*}-\gamma_{i} P_{i}\left(\left|x_{*}-x\right|\right)\left(x_{*}-x\right)+\eta_{i 2} D_{i}\left(x_{*}\right) .
\end{aligned}
$$

This collision mechanism (26)-(27) is directly inspired from [77], where there is only one group (of followers). On the other hand, the interaction between a follower with opinion $x$ and an opinion leader with opinion $x_{*}$ gives postcollisional opinions

$$
\begin{aligned}
x^{\prime} & =x-\gamma_{3} P_{3}\left(\left|x-x_{*}\right|\right)\left(x-x_{*}\right)+\eta_{11} D_{1}(x), \\
x_{*}^{\prime} & =x_{*} .
\end{aligned}
$$

The update of a follower's opinion (28) is exactly the same as in (26). On the contrary, as stated in (29), a leader's opinion does not evolve at all during an interaction with a follower.

We refer to 3.1 for the meaning of $\gamma_{k}, P_{k}, \eta_{i j}$ and $D_{j}$, for $k=1,2,3$ and $i, j=1,2$.

The distribution functions $f_{i}, i=1,2$, are then governed by the system of two Boltzmann-like equations

$$
\begin{aligned}
& \frac{\partial f_{1}}{\partial t}=\frac{1}{\tau_{11}} Q_{11}\left(f_{1}, f_{1}\right)+\frac{1}{\tau_{12}} Q_{12}\left(f_{1}, f_{1}\right), \\
& \frac{\partial f_{2}}{\partial t}=\frac{1}{\tau_{22}} Q_{22}\left(f_{2}, f_{2}\right),
\end{aligned}
$$

where $\tau_{i j}$ are the relaxation times, and the collision operators write, under their weak form,

$$
\begin{aligned}
& \int_{-1}^{1} Q_{i j}\left(f_{i}, f_{j}\right)(x, t) \phi(x) \mathrm{d} x \\
= & \frac{1}{2}\left\langle\iint_{(-1,1)^{2}}\left[\phi\left(x_{*}^{\prime}\right)+\phi\left(x^{\prime}\right)-\phi\left(x_{*}\right)-\phi(x)\right] f_{i}(x, t) f_{j}\left(x_{*}, t\right) \mathrm{d} x \mathrm{~d} x_{*}\right\rangle,
\end{aligned}
$$

for all smooth enough test functions $\phi$.

Starting from microscopic interactions among individuals, the authors also obtain a quasi-invariant limit, described by a system of Fokker-Planck-type equations, and discuss its steady states.

\subsection{Political plurality}

The political plurality is one of the main characteristics of our societies. Despite the very theoretical viewpoint that the media should be totally indepedent from the political class, it is clear that both are deeply interconnected. 
Besides, this topic is quite difficult to handle. Indeed, as we can see in the next paragraphs, the models which are used here have, for each party, either several distribution functions or a multidimensional opinion vector. The fact that there are few works (see [43] for example) about that too is quite meaningful.

\section{Propaganda and politicians}

Following [61], in [62], Lo Schiavo designs a specific model to describe the dynamics of a composite, structured society where there are two competing political parties. The model structure once again contains terms with localized interactions and mean field terms. The variable of the standard population distribution function is again the social state $u$, but, this time, the population division is more intricate. There are now two categories. The first one is constituted of electors, who can be split into three subgroups: party 1, party 2 and the opinionless people regarding the parties. The second one is the political class itself. Its associated variable can be seen as the ideological position $\nu \in[-1,+1]$, which very much looks alike an opinion from the left $(-1)$ to the right wing $(+1)$. We must emphasize that the ideological position variable of the political class is not at all linked to the social state of the electors. Nevertheless, they may be treated in the same way. Hence, the system which is eventually obtained now is very similar to (6). Indeed, if we denote by $f_{0}$ the density function for the political class, by $f_{1}$ and $f_{2}$ the ones for the electors respectively favouring party 1 and 2 , by $f_{3}$ the one for nonvoting electors, and, again, by $\boldsymbol{f}=\left(f_{i}\right)_{0 \leq i \leq 3}$, Lo Schiavo can write, for any $t, u$ and $\nu$,

$$
\begin{aligned}
& \frac{\partial f_{i}}{\partial t}(t, u)+\frac{\partial\left(f_{i} K_{i}(\boldsymbol{f})\right)}{\partial u}(t, u)=Q_{i}(\boldsymbol{f})(t, u)+R_{i}(\boldsymbol{f})(t, u), \quad 1 \leq i \leq 3, \\
& \frac{\partial f_{0}}{\partial t}(t, \nu)+\frac{\partial\left(f_{0} K_{0}(\boldsymbol{f})\right)}{\partial \nu}(t, \nu)=S_{0}(\boldsymbol{f})(t, \nu) .
\end{aligned}
$$

The functions $K_{i}$ are again the propaganda functions, whose sociological meaning is discussed in [54]. The operators $Q_{i}$ have the same meaning as in (6), they model the microscopic interactions between electors. The operators $R_{i}$ model the effect of the political class on the electors, and $S_{0}$ takes into account the interactions inside the political class.

Eventually, in [63], Lo Schiavo presents a reduced version of the previous system (33)-(34). The model structure still contains terms with localized interactions and mean field terms. The main strength of this work is to emphasize the influence of some terms which were only briefly discussed in [62].

\section{Mass media and multipartite situation}

In [14], the authors propose a kinetic model to describe the evolution of the opinion in a closed group with respect to a choice between multiple options, such as political parties. Two main mechanisms of opinion formation are taken 
into account: the binary interaction between individuals, as in [15], and the effects of the mass media.

In multi-choice situations, a major problem consists in the fact that, in general, it is not possible to rank the options independently on the individual. The authors introduce therefore an opinion vector, whose dimension coincides with the number of possible choices. If there exist $p \geq 1$ options of choice, $x_{i} \in[-1,1]$ is the agreement variable associated to the choice $P_{i}, 1 \leq i \leq p$, and the opinion (or agreement) vector $\boldsymbol{x}=\left(x_{1}, \ldots, x_{p}\right) \in[-1,1]^{p}$ gives, for each individual of the population, its opinion about the plurality of options. Nevertheless, the distribution function $f$, defined on $\mathbb{R}_{+} \times[-1,1]^{p}$, is still one-dimensional.

The first phenomenon taken into account is the binary exchange of opinions inside the population: if $\boldsymbol{x}, \boldsymbol{x}^{*}$ are the opinion vectors of two individuals before an interaction, the post-collisional opinions are obtained through a generalization of the collision rule defined in [15]:

$$
\left\{\begin{array}{l}
x_{i}^{\prime}=\frac{x_{i}+x_{i}^{*}}{2}+\eta\left(x_{i}\right) \frac{x_{i}-x_{i}^{*}}{2} \\
\left(x_{i}^{*}\right)^{\prime}=\frac{x_{i}+x_{i}^{*}}{2}+\eta\left(x_{i}^{*}\right) \frac{x_{i}^{*}-x_{i}}{2}
\end{array} \quad 1 \leq i \leq p .\right.
$$

Once defined the collision rule (35), the interaction between individuals and the corresponding exchange of opinions is described by a collisional integral of Boltzmann type. The weak form of the collision kernel is

$$
\langle Q(f, f), \varphi\rangle=\iint_{(-1,1)^{2 p}} \beta\left(\boldsymbol{x}, \boldsymbol{x}^{*}\right) f(t, \boldsymbol{x}) f\left(t, \boldsymbol{x}^{*}\right)\left[\varphi\left(\boldsymbol{x}^{\prime}\right)-\varphi(\boldsymbol{x})\right] \mathrm{d} \boldsymbol{x}^{*} \mathrm{~d} \boldsymbol{x},
$$

where $\varphi$ is a smooth enough test function in the variable $\boldsymbol{x}$, and $\beta:[-1,1]^{2} \rightarrow$ $\mathbb{R}_{+}$is the cross section, which depends on a suitable pre-collisional opinion distance.

The effects of the media on the population are modelled by a background, which can be compared to the background introduced in [25]. This assumption adds a linear kinetic term into the equation. For any media $M_{j}, 1 \leq j \leq m$, the authors introduce two quantities: its strength $\alpha_{j}$, which translates the influence of the media on the population and its opinion vector $\boldsymbol{X}^{j} \in[-1,1]^{p}$, with respect to each option of choice.

The effect of each media $M_{j}$ on the individual is therefore described by an interaction rule which reminds the collision rule (35):

$$
\tilde{x_{i}}=x_{i}+\xi_{j}\left(\left|X_{i}^{j}-x_{i}\right|\right)\left(X_{i}^{j}-x_{i}\right),
$$

for all $i$ and $j$. The functions $\xi_{j}$ are the microscopic media attraction functions. The influence of each media is then described by a (possibly time-dependent) linear integral operator, $L_{j}, 1 \leq j \leq m$, that has the classical structure of the linear Boltzmann kernels (see 2.2). Its weak form is 


$$
\left\langle L_{j} f, \varphi\right\rangle=\alpha_{j} \int_{(-1,1)^{p}} f(t, \boldsymbol{x})[\varphi(\tilde{\boldsymbol{x}})-\varphi(\boldsymbol{x})] \mathrm{d} \boldsymbol{x},
$$

where $\varphi=\varphi(\boldsymbol{x})$ is a suitably regular test function.

By combining the two phenomena, the evolution law of the unknown $f$ is the following integro-differential equation of kinetic type, written in the distributional sense,

$$
\frac{\partial f}{\partial t}=\sum_{j=1}^{m} L_{j} f+Q(f, f),
$$

The authors prove the existence and uniqueness of the solution to (36)-(38) in $C^{0}\left([0, T] ; L^{1}\left((-1,1)^{p}\right)\right)$ for initial data in $L^{1}\left((-1,1)^{p}\right.$, and provide some numerical tests. In particular, if they use time dependent $\boldsymbol{X}^{j}$, they also obtain time dependent behaviours for the parts of the population in favour of each party. That means that we may not obtain a relevant asymptotic behaviour of the distribution function.

\section{Conclusion}

In this chapter, we had the opportunity to describe numerous kinetic models fitted to the study of opinion dynamics. We pointed out that the main feature of the opinion formation process is the tendency to compromise, and we recovered it in all the models, obtaining Boltzmann-like equations. We also presented models taking into account the self-thinking, the voting process, the presence of contradictory people or leaders in the society, the propaganda through the media, and a multipartite democracy. We gave some tracks about quasi-invariant limits for those models, leading to Fokker-Planck equations.

In many papers we reviewed, some numerical results are given, often regarding simple situations of interest. Those results allow to emphasize the main features of the models under study. We invite the reader to refer to the articles themselves to check that numerical part of the works. The numerical schemes or methods are discussed there, in link with our section dedicated to the numerical methods to solve the Boltzmann equations 2.3.

As we already pointed out in 1.1, there are lots of theoretical contributions, but not so many with sociophysical data. In fact, the auxiliary functions and coefficients are not really investigated from a sociological point of view. Three of the articles we reviewed in this contribution tried to open the road. Helbing [48] worked on the German migration data given in [80]: having the state $y \in\{1, \ldots, S\}$ means "living in a region $y$ ", where $S$ was the number of West Germany regions. In [5], the authors discuss their model around the 1993 federal elections in Canada. Eventually, in [31], the authors test the behaviour of their model by confronting them to the results of the state elections in Carinthia (Austria). To enhance the models we investigate, we should try to systematically confront them with real data, such as existing polls, and then obtain more and more realistic models for the opinion formation. 


\section{References}

1. G. Aletti, G. Naldi, and G. Toscani. First-order continuous models of opinion formation. SIAM J. Appl. Math., 67(3):837-853 (electronic), 2007.

2. L. Arlotti, N. Bellomo, and E. De Angelis. Generalized kinetic (Boltzmann) models: mathematical structures and applications. Math. Models Methods Appl. Sci., 12(4):567-591, 2002.

3. E. Ben-Naim. Opinion dynamics: rise and fall of political parties. Europhys. Lett., 69:671-677, 2005.

4. E. Ben-Naim, P. L. Krapivsky, and S. Redner. Bifurcation and patterns in compromise processes. Phys. D, 183(3-4):190-204, 2003.

5. E. Ben-Naim, P. L. Krapivsky, F. Vazquez, and S. Redner. Unity and discord in opinion dynamics. Phys. A, 330(1-2):99-106, 2003. Randomness and complexity (Eilat, 2003).

6. M.-L. Bertotti and M. Delitala. On the qualitative analysis of the solutions of a mathematical model of social dynamics. Appl. Math. Lett., 19(10):1107-1112, 2006.

7. M.-L. Bertotti and M. Delitala. Conservation laws and asymptotic behavior of a model of social dynamics. Nonlinear Anal. Real World Appl., 9(1):183-196, 2008.

8. M.-L. Bertotti and M. Delitala. On a discrete generalized kinetic approach for modelling persuader's influence in opinion formation processes. Math. Comput. Modelling, 48(7-8):1107-1121, 2008.

9. M.-L. Bertotti and M. Delitala. On the existence of limit cycles in opinion formation processes under time periodic influence of persuaders. Math. Models Methods Appl. Sci., 18(6):913-934, 2008.

10. G.A. Bird. Molecular gas dynamics and the direct simulation of gas flows, volume 42 of Oxford Engineering Science Series. The Clarendon Press Oxford University Press, New York, 1995. Corrected reprint of the 1994 original.

11. L. Boltzmann. Weitere studien über das wärmegleichgewicht unter gasmolekülen. Sitzungsberichte Akad. Wiss., 66:275-370, 1873.

12. L. Boltzmann. Lectures on gas theory. Translated by Stephen G. Brush. University of California Press, Berkeley, 1964.

13. L. Boudin, A. Mercier, and F. Salvarani. Conciliatory and contradictory dynamics in opinion formation. Technical report, Lab. J.-L. Lions, UPMC, 2009.

14. L. Boudin, R. Monaco, and F. Salvarani. A kinetic model for multidimensional opinion formation. Technical report, Lab. J.-L. Lions, UPMC, 2009.

15. L. Boudin and F. Salvarani. A kinetic approach to the study of opinion formation. M2AN Math. Model. Numer. Anal., 43(3):507-522, 2009.

16. L. Boudin and F. Salvarani. The quasi-invariant limit for a kinetic model of sociological collective behavior. Kinet. Relat. Models, 2(3):433-449, 2009.

17. C. Buet, S. Cordier, and P. Degond. Regularized Boltzmann operators. Comput. Math. Appl., 35(1-2):55-74, 1998. Simulation methods in kinetic theory.

18. M. Campiti, G. Metafune, and D. Pallara. Degenerate self-adjoint evolution equations on the unit interval. Semigroup Forum, 57(1):1-36, 1998.

19. K.M. Case and P.F. Zweifel. Linear transport theory. Addison-Wesley Publishing Co., Reading, Mass.-London-Don Mills, Ont., 1967.

20. C. Castellano, S. Fortunato, and V. Loreto. Statistical physics of social dynamics. Rev. Mod. Phys., 81:591-646, 2009. 
21. C. Cercignani. The Boltzmann equation and its applications, volume 67 of Applied Mathematical Sciences. Springer-Verlag, New York, 1988.

22. C. Cercignani, R. Illner, and M. Pulvirenti. The mathematical theory of dilute gases, volume 106 of Applied Mathematical Sciences. Springer-Verlag, New York, 1994.

23. S. Chandrasekhar. Radiative transfer. Dover Publications Inc., New York, 1960.

24. W. Cholewa. Aggregation of fuzzy opinions - an axiomatic approach. Fuzzy Sets and Systems, 17(3):249-258, 1985.

25. V. Comincioli, L. Della Croce, and G. Toscani. A Boltzmann-like equation for choice formation. Kinet. Relat. Models, 2(1):135-149, 2009.

26. G. Deffuant, D. Neau, F. Amblard, and G. Weisbuch. Mixing beliefs among interacting agents. Adv. Complex Systems, 3:87-98, 2000.

27. P. Degond and S. Mas-Gallic. The weighted particle method for convectiondiffusion equations. I. The case of an isotropic viscosity. Math. Comp., 53(188):485-507, 1989.

28. P. Degond and S. Mas-Gallic. The weighted particle method for convectiondiffusion equations. II. The anisotropic case. Math. Comp., 53(188):509-525, 1989.

29. P. Degond, L. Pareschi, and G. Russo, editors. Modeling and computational methods for kinetic equations. Modeling and Simulation in Science, Engineering and Technology. Birkhäuser Boston Inc., Boston, MA, 2004.

30. L. Desvillettes and F. Salvarani. Characterization of collision kernels. M2AN Math. Model. Numer. Anal., 37(2):345-355, 2003.

31. B. Düring, P. Markowich, J.-F. Pietschmann, and M.-T. Wolfram. Boltzmann and Fokker-Planck equations modelling opinion formation in the presence of strong leaders. Proc. R. Soc. A, 465(2112):2687-3708, 2009.

32. M. Fansten. Introduction à une théorie mathématique de l'opinion. Ann. I.N.S.E.E., 21:3-55, 1976.

33. S. French. Updating of belief in the light of someone else's opinion. J. Roy. Statist. Soc. Ser. A, 143(1):43-48, 1980.

34. S. Galam. Contrarian deterministic effects on opinion dynamics: "the hung elections scenario". Phys. A, 333(1-4):453-460, 2004.

35. S. Galam. Stability of leadership in bottom-up hierarchical organizations. $J$. Soc. Complex., 2(2):62-75, 2006.

36. S. Galam. From 2000 Bush-Gore to 2006 Italian elections: voting at fifty-fifty and the contrarian effect. Qual. Quant., 41(4):579-589, 2007.

37. S. Galam. Sociophysics: a review of Galam models. Int. J. Mod. Phys. C, 19(4):409-440, 2008.

38. S. Galam, Y. Gefen, and Y. Shapir. Sociophysics: a new approach of sociological collective behaviour. i. Mean-behaviour description of a strike. J. Math. Sociol., 9:1-23, 1982.

39. S. Galam and S. Moscovici. Towards a theory of collective phenomena: consensus and attitude changes in groups. Eur. J. Soc. Psychol., 21:49-74, 1991.

40. S. Galam and J.-D. Zucker. From individual choice to group decision-making. Phys. A, 287(3-4):644-659, 2000. Economic dynamics from the physics point of view (Bad Honnef, 2000).

41. G. Gallavotti. Rigorous theory of the boltzmann equation in the lorentz gas. Technical report, Nota interna n. 358, Istituto di Fisica, Università di Roma, 1973. 
42. R. Gatignol. Théorie cinétique des gaz à répartition discrète de vitesses. Springer Verlag, Berlin, 1975.

43. S. Gekle, L. Peliti, and S. Galam. Opinion dynamics in a three-choice system. Eur. Phys. J. B, 45:569-575, 2005.

44. C. Genest, S. Weerahandi, and J. V. Zidek. Aggregating opinions through logarithmic pooling. Theory and Decision, 17(1):61-70, 1984.

45. D.B. Goldstein. Discrete-velocity collision dynamics for polyatomic molecules. Phys. Fluids A, 4(8):1831-1839, 1992.

46. D. Helbing. Boltzmann-like and Boltzmann-Fokker-Planck equations as a foundation of behavioral models. Phys. A, 196:546-573, 1993.

47. D. Helbing. Stochastic and Boltzmann-like models for behavioral changes, and their relation to game theory. Phys. A, 193:241-258, 1993.

48. D. Helbing. A mathematical model for the behavior of individuals in a social field. J. Math. Sociol., 19(3):189-219, 1994.

49. A. B. Huseby. Combining opinions in a predictive case. In Bayesian statistics, 3 (Valencia, 1987), Oxford Sci. Publ., pages 641-651. Oxford Univ. Press, New York, 1988.

50. R. Illner and M. Pulvirenti. Global validity of the Boltzmann equation for a two-dimensional rare gas in vacuum. Comm. Math. Phys., 105(2):189-203, 1986.

51. R. Illner and M. Pulvirenti. Global validity of the Boltzmann equation for two- and three-dimensional rare gas in vacuum. Erratum and improved result. Comm. Math. Phys., 121(1):143-146, 1989.

52. T. Inamuro and B. Sturtevant. Numerical study of discrete-velocity gases. Phys. Fluids A, 2(12):2196-2203, 1990.

53. E. Jäger and L.A. Segel. On the distribution of dominance in populations of social organisms. SIAM J. Appl. Math., 52(5):1442-1468, 1992.

54. G.S. Jowett and V. O'Donnell. Propaganda and persuasion. Sage publications, fourth edition, 2005.

55. M. Kac. Probability and related topics in physical sciences, volume 1957 of With special lectures by G. E. Uhlenbeck, A. R. Hibbs, and B. van der Pol. Lectures in Applied Mathematics. Proceedings of the Summer Seminar, Boulder, Colo. Interscience Publishers, London-New York, 1959.

56. B. K. Kale. On tests of hypotheses regarding the change of opinions. Ann. Soc. Sci. Bruxelles Sér. I, 88:305-312, 1974.

57. S. Kaniel and M. Shinbrot. The Boltzmann equation. II. Some discrete velocity models. J. Mécanique, 19(3):581-593, 1980.

58. O.E. Lanford III. Time evolution of large classical systems. In Dynamical systems, theory and applications (Recontres, Battelle Res. Inst., Seattle, Wash., 1974), pages 1-111. Lecture Notes in Phys., Vol. 38. Springer, Berlin, 1975.

59. T.M. Liggett. Stochastic interacting systems: contact, voter and exclusion processes, volume 324 of Grundlehren der Mathematischen Wissenschaften [Fundamental Principles of Mathematical Sciences]. Springer-Verlag, Berlin, 1999.

60. M. Lo Schiavo. Population kinetic models for social dynamics: dependence on structural parameters. Comput. Math. Appl., 44(8-9):1129-1146, 2002.

61. M. Lo Schiavo. The modelling of political dynamics by generalized kinetic (Boltzmann) models. Math. Comput. Modelling, 37(3-4):261-281, 2003.

62. M. Lo Schiavo. Kinetic modelling and electoral competition. Math. Comput. Modelling, 42(13):1463-1486, 2005.

63. M. Lo Schiavo. A dynamical model of electoral competition. Math. Comput. Modelling, 43(11-12):1288-1309, 2006. 
64. E. Marchi. A game-theoretical approach to some situations in opinion making. Math. Biosci., 2:85-109, 1968.

65. R. Monaco and L. Preziosi. Fluid dynamic applications of the discrete Boltzmann equation, volume 3 of Series on Advances in Mathematics for Applied Sciences. World Scientific Publishing Co. Inc., River Edge, NJ, 1991.

66. F. J. Montero de Juan. A note on Fung-Fu's theorem. Fuzzy Sets and Systems, $17(3): 259-269,1985$.

67. F. J. Montero de Juan. Aggregation of fuzzy opinions in a nonhomogeneous group. Fuzzy Sets and Systems, 25(1):15-20, 1988.

68. K. Nanbu. Direct simulation scheme derived from the Boltzmann equation. I. Monocomponent gases. J. Phys. Soc. Jpn., 49(5):2042-2049, 1980.

69. K. Nanbu. Direct simulation scheme derived from the Boltzmann equation. II. Multicomponent gas mixtures. J. Phys. Soc. Jpn., 49(5):2050-2054, 1980.

70. T. Platkowski and R. Illner. Discrete velocity models of the Boltzmann equation: a survey on the mathematical aspects of the theory. SIAM Rev., 30(2):213-255, 1988.

71. M. Pulvirenti. Global validity of the Boltzmann equation for a three-dimensional rare gas in vacuum. Comm. Math. Phys., 113(1):79-85, 1987.

72. F. Rogier and J. Schneider. A direct method for solving the Boltzmann equation. Transport Theory Statist. Phys., 23(1-3):313-338, 1994.

73. F. Slanina and H. Lavička. Analytical results for the Sznajd model of opinion formation. Eur. Phys. J. B, 35:279-288, 2003.

74. E. Sonnendrücker, J. Roche, P. Bertrand, and A. Ghizzo. The semi-Lagrangian method for the numerical resolution of the Vlasov equation. J. Comput. Phys., 149(2):201-220, 1999.

75. V. Sood, T. Antal, and S. Redner. Voter models on heterogeneous networks. Phys. Rev. E, 77(4):041121, 2008.

76. K. Sznajd-Weron and J. Sznajd. Opinion evolution in closed community. Int. J. Mod. Phys. C, 11:1157-1166, 2000.

77. G. Toscani. Kinetic models of opinion formation. Commun. Math. Sci., 4(3):481-496, 2006.

78. C. Villani. A review of mathematical topics in collisional kinetic theory. In Handbook of mathematical fluid dynamics, Vol. I, pages 71-305. North-Holland, Amsterdam, 2002.

79. W. Weidlich. The statistical description of polarization phenomena in society. Br. J. Math. Stat. Psychol., 24:251-266, 1971.

80. W. Weidlich and G. Haag, editors. Interregional migration: dynamic theory and comparative analysis. Springer-Verlag, Berlin, 1988.

81. E. Wild. On Boltzmann's equation in the kinetic theory of gases. Proc. Cambridge Philos. Soc., 47:602-609, 1951. 